\title{
Exclude Body Weight Data From Statistics Indicator
}

National Cancer Institute

\section{Source}

National Cancer Institute. Exclude Body Weight Data From Statistics Indicator. NCI

Thesaurus. Code C119783.

Specifies whether the body weight result values should be excluded from the statistical analysis. 